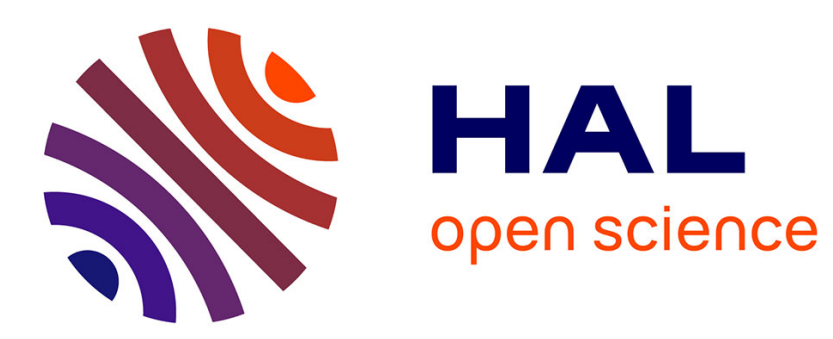

\title{
Floc Cohesive Force in Reversible Aggregation: A Couette Laminar Flow Investigation
}

\author{
G. Frappier, B. Lartiges, S. Skali-Lami
}

\section{To cite this version:}

G. Frappier, B. Lartiges, S. Skali-Lami. Floc Cohesive Force in Reversible Aggregation: A Couette Laminar Flow Investigation. Langmuir, 2010, 26 (13), pp.10475-10488. 10.1021/la9046947 . hal02187124

\section{HAL Id: hal-02187124 \\ https://hal.science/hal-02187124}

Submitted on 17 Jul 2019

HAL is a multi-disciplinary open access archive for the deposit and dissemination of scientific research documents, whether they are published or not. The documents may come from teaching and research institutions in France or abroad, or from public or private research centers.
L'archive ouverte pluridisciplinaire HAL, est destinée au dépôt et à la diffusion de documents scientifiques de niveau recherche, publiés ou non, émanant des établissements d'enseignement et de recherche français ou étrangers, des laboratoires publics ou privés. 


\title{
Floc cohesive force in reversible aggregation: A Couette laminar flow investigation
}

\author{
G. Frappier ${ }^{1}$, B.S. Lartiges ${ }^{1 *}$, S. Skali-Lami ${ }^{2 *}$ \\ ${ }^{1}$ Nancy University - LEM-ENSG/INPL-CNRS, Pôle de l'Eau 15 Avenue du Charmois - BP 40 \\ 54501 Vandoeuvre Cedex, FRANCE \\ ${ }^{2}$ Nancy University - LEMTA-ENSEM/INPL-CNRS, 2 Avenue de la Forêt de Haye - BP 160 \\ 54504 Vandoeuvre Cedex, FRANCE \\ E-mail: bruno. lartiges@get.omp.eu \\ salah.skali-lamidensem.inpl-nancy.fr
}

(*) To whom correspondence should be addressed 


\begin{abstract}
A simple theoretical model is proposed to describe the limiting size of aggregates attained at steady-state under given shear conditions. The stable size is assumed to be the result of a dynamic equilibrium between simultaneous aggregate growth and breakup that are represented as first-order processes. The theory establishes that the evolution of steady-state aggregate size versus shear rate is written as the sum of two exponential laws. The validity of the model is verified by direct observation of the coagulation behavior of latex particles in the stagnant plane of a counterrotating Couette reactor. The influence of latex elementary particle size, initial particle volume fraction, and inner gap spacing of Couette reactor, are investigated. In all cases, the model shows good agreement with the experimental results. Aggregate growth proceeds with a monomodal size distribution that exhibits a scaling behavior. Such monomodal distribution evolves towards broad and even bimodal steady-state distributions at both low and high shear rates, whereas a narrow monomodal pattern is observed at intermediate shear gradients. The aggregate cohesive force $F_{H}$ can calculated from the critical shear rate of dislocation defined by the model. In contrast to the broadly accepted view that larger flocs should be more fragile than smaller aggregates, we find that $\mathrm{F}_{\mathrm{H}}$ scales as $\mathrm{D}^{3 / 2}$ where $\mathrm{D}$ is the aggregate characteristic diameter. The latter relationship may be derived by assuming linear elasticity of aggregates.
\end{abstract}




\section{INTRODUCTION}

Floc binding strength is considered as a key-parameter in most solid/liquid separation processes involving the formation of aggregates under agitation ${ }^{1}$. At first approximation, it represents the ability of aggregated particles to resist breakup under hydrodynamic shearing forces. However, the way hydrodynamic stresses are applied onto a floc, the irregular shape and the structure of aggregates, the kind of particle-particle association reached within the floc (strength of inter-particle bond and number of individual bonds between primary particles), imply that the cohesive force of aggregates is actually the result of overlapping and interdependent physical phenomena.

A floc binding strength can nevertheless be directly determined by pulling apart individual aggregates using a micromechanics approach ${ }^{2,3}$. Rupture forces in the 20-200 nN range were thus measured for calcium carbonate particles flocculated with polymers with no particular dependence of aggregate cohesive force on floc size. The breakup behaviour of flocs can also be investigated by subjecting preformed aggregates to well-defined flow fields such as four-roller extensional flow ${ }^{4-6}$, contraction flow $^{6-9}$ or turbulent flow ${ }^{10,68}$. A quantitative estimate of floc strength $F_{C}$ is then obtained by relating the aggregate size at which rupture is observed to the local hydrodynamic shear forces (e.g. $F_{C} \sim \mu G^{2}$ under pure shear flow with $\mathrm{G}$ the velocity gradient, $\mathrm{D}$ a characteristic aggregate size, and $\mu$ the dynamic viscosity). Typically, coagulated systems yield cohesive forces of about $2 \mathrm{nN}$, whereas flocculated systems are characterized by values of floc strength one to two orders of magnitude higher ${ }^{9,10}$. Interestingly, the critical force required to induce floc breakup was found to increase with aggregate size for kaolin particles flocculated with either aluminium sulfate, ferric chloride, or polyacrylamide polymer ${ }^{10,11}$ and for silica particles aggregated with ferric chloride . $^{6}$

In the general case when the fluid motions can not be precisely specified, the floc strength is evaluated indirectly by monitoring a characteristic size D of aggregates formed under variable shear conditions ${ }^{12}$. Indeed, for suspensions coagulated in the presence of an excess electrolyte at a given shear rate, a steady-state floc size is reached after a certain time, such stable size being smaller and attained more rapidly with an increase in shear rate ${ }^{12,13}$. The decrease in steady-state floc size $D$ as a function of the applied shear rate $G$ follows the empirical expression:

$$
\mathrm{D}=\alpha \mathrm{G}^{-\beta}
$$


in which $\alpha$ and $\beta$ are constants. For colloidal dispersions destabilized with polymers or hydrolyzing metal salts, equation (1) is also applied even though the aggregation kinetics is more complex with a frequent overshoot followed by a continuous decrease in aggregate $\operatorname{size}^{14-16}$.

The experimental values of $\beta$ reported in the literature vary between 0.3 and $0.8{ }^{17}$. Over the past four decades, numerous attempts have been made to model aggregate rupture in agitated reactors, and hence to provide a theoretical basis to equation (1) ${ }^{18-23}$. According to the nature of hydrodynamic forces acting on the aggregate, the breakage mechanism of flocs, i.e. erosion of elementary particles/small floc subunits from the outer part of aggregates or fragmentation of flocs into large-scale pieces, the type of particle-particle connecting bonds within the aggregate, and the description of floc structure, values ranging between 0.2 to 1 have been proposed for the exponent $\beta$. In most cases, the stable floc size reached under agitation has been interpreted from a simple mechanical approach, i.e. a force balance between aggregate strength and hydrodynamic stresses exerted onto the floc. However, the limiting size may also represent (i) a dynamic equilibrium between simultaneous growth and breakup of aggregates ${ }^{24}$, (ii) a decrease in aggregation efficiency as a function of floc size $\mathrm{e}^{25}$. These two dynamic aspects, generally overlooked in previous aggregate breakup modeling, certainly participate to the definition of the steady-state floc size.

The objectives of this paper are then (i) to provide a theoretical description of stable floc size that takes into account the dynamic nature of the aggregation process; the model introduces a critical shear rate of aggregate breakup that enables the calculation of the floc cohesive force. (ii) To verify experimentally the efficacy of our model; this involved the insitu measurements of aggregate size in the laminar flow generated within a counterrotating cylinder Couette device.

\section{THEORETICAL CONSIDERATION}

Under dynamic steady-state conditions, we assume that (i) shear aggregation is predominant and Brownian motion insignificant, (ii) floc growth and floc breakup can be described as first-order processes (which is consistent with the formalisms developed in nonequilibrium thermodynamics close to the stationary state), (iii) aggregate plastic deformation is considered negligible during a breakup or a collision event, (iv) the aggregated system can 
be represented by a characteristic size D. The limiting floc size at a given shear rate $\dot{\gamma}$ can then be expressed by:

$$
D(\dot{\gamma}+d \dot{\gamma})=D(\dot{\gamma})-\frac{D(\dot{\gamma})}{\tau_{d}} d \tau+\frac{1}{G_{F}}\left[\int_{\dot{\gamma}}^{0} \frac{D(\zeta)-D_{c}}{G_{F}} d \zeta\right] d \dot{\gamma}
$$

where ${ }_{d}$ is the critical shear stress that must be applied to determine floc dislocation, $G_{f}$ the critical shear gradient for inducing floc formation, and $D_{c}$ the characteristic size of aggregated subunits that participate to the floc growth. The second term on the right-hand side of equation (2) accounts for the loss of aggregated matter due to break-up, whereas the last term denotes the gain of aggregated matter due to floc growth. The integral represents the shear history that generated the aggregate of characteristic size D at shear rate $\dot{\gamma}$. Since the model focuses on the definition of a steady-state floc size, neither the detailed aggregate structure, nor the local hydrodynamic interactions between colliding flocs, need to be explicitly described. It should be noted that equation (1) becomes a solution to (2) if the term representing floc formation is dropped.

Differentiating twice equation (2), yields:

$$
\frac{d^{2} D(\dot{\gamma})}{d \dot{\gamma}^{2}}=-\frac{d D(\dot{\gamma})}{d \dot{\gamma}} \frac{d \tau}{d \dot{\gamma}} \frac{1}{\tau_{d}}-\frac{D(\dot{\gamma})}{\tau_{d}} \frac{d^{2} \tau}{d \dot{\gamma}^{2}}-\frac{D(\dot{\gamma})}{G_{F}^{2}}
$$

As a first approximation, we assume that the floc volume fraction remains sufficiently low to consider that the coagulated suspension behaves as a Newtonian fluid. In other terms, $\mu$ is constant and hence, $\frac{d \tau}{d \dot{\gamma}}=\mu$ and $\frac{\mathrm{d}^{2} \tau}{d \dot{\gamma}^{2}}=0$. Substituting into (3) leads to:

$$
\frac{d^{2} D(\dot{\gamma})}{d \dot{\gamma}^{2}}=-\frac{d D(\dot{\gamma})}{d \dot{\gamma}} \frac{\mu}{\tau_{d}}-\frac{D(\dot{\gamma})}{G_{F}^{2}}
$$

which analytical solution is:

$$
D(\dot{\gamma})=a \mathrm{e}^{\frac{\dot{\gamma}}{2}\left(-\frac{\mu}{\tau_{d}}-\sqrt{\left(\frac{\mu}{\tau_{d}}\right)^{2}-4\left(\frac{1}{G_{F}}\right)^{2}}\right)}+\mathrm{b} \mathrm{e}^{\frac{\dot{\gamma}}{2}\left(-\frac{\mu}{\tau_{d}}+\sqrt{\left(\frac{\mu}{\tau_{d}}\right)^{2}-4\left(\frac{1}{G_{F}}\right)^{2}}\right)}
$$

$\mathrm{a}$ and $\mathrm{b}$ being two arbitrary integration constants. Therefore, $\mathrm{D}(\dot{\gamma}=0)=\mathrm{a}+\mathrm{b}=\mathrm{D}_{\max } . \mathrm{G}_{\mathrm{F}}, \tau_{\mathrm{d}}$, a, and $b$, are obtained by adjusting equation (5) to experimental data. Finally, taking into acount both floc formation and breakup in the definition of a steady-state aggregate size, implies that the evolution of stable floc diameter versus shear rate can be written as the sum of two exponential laws. 


\section{EXPERIMENTAL SECTION}

Suspension preparation. Polystyrene latex particles carrying a slight anionic charge from sulfate ester groups, were purchased from Polysciences Inc. The mean particle diameters determined by dynamic light scattering (Malvern PCS 4800), were $0.202 \mu \mathrm{m} \pm 2 \%$ and $2 \mu \mathrm{m}$ $\pm 3 \%$. The aggregates were prepared by diluting an aliquot of latex stock suspension with appropriate amounts of deionized water (Milli-Q Millipore) and aqueous 2.9 M NaCl solution (Aldrich $99.7 \%$ ) to yield solid volume fractions $(\phi)$ of either $510^{-5}, 10^{-4}$ or $10^{-3}$, and a final $1.45 \mathrm{M} \mathrm{NaCl}$ concentration that matches the specific gravity of latex particles $\left(1.05 \mathrm{~g} / \mathrm{cm}^{3}\right)$. Centrifugal forces and differential settling can then be neglected during the experiments. Both deionized water and $\mathrm{NaCl}$ solution were preliminarily filtered with a $0.22 \mu \mathrm{m}$ pore size cellulose-acetate membrane (porafil@ Macherey-Nagel), and placed in a thermostated bath set at $30^{\circ} \mathrm{C}$. The $\mathrm{pH}$ of the salt solution was adjusted to 7.0 using $\mathrm{NaOH} 0.1 \mathrm{M}$. The aggregating suspension was then let to reach thermal equilibrium in the thermostated bath for further 30 minutes prior to its introduction in the Couette device.

Couette-flow reactor and aggregation procedure. The shear aggregation experiments were conducted in a Couette-flow reactor in which both the inner and the outer cylinders can rotate in same or in opposite directions (fig. 1a). The outer cylinder had an internal diameter of 10 $\mathrm{cm}$ and a height of $25 \mathrm{~cm}$. Two gap spacings, $\mathrm{e}=1.5 \mathrm{~mm}$ and $\mathrm{e}=3 \mathrm{~mm}$, were used to investigate the effect of confinement on aggregate characteristics. Each cylinder was rotated by a precision motor (Maxon A-max PLG32) connected to a digital controller (Encoder HEDL-5540). In the present work, the inner and outer cylinder angular frequencies $\Omega_{1}$ and $\Omega_{2}$ were selected such that $\Omega_{1}=-\Omega_{2}=\Omega$. A stagnation plane is then created halfway of cylinders. In cylindrical coordinates, the shear rate $\dot{\gamma}(\mathrm{r})$ at a given radial distance $\mathrm{r}$ within the gap can be determined from ${ }^{26}$ :

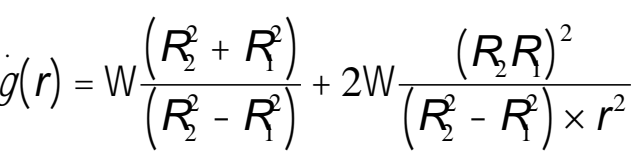

where $R_{1}$ and $R_{2}$ are the radii of the inner and outer cylinders, respectively. The variation in $\dot{\gamma}$ with $\mathrm{r}$ inside the gap is about $\pm 3 \%$. Typical $\dot{\gamma}(\mathrm{r})$ profiles determined at various angular velocities are shown in figure $1 \mathrm{~b}$. The spatial mean shear rate $\mathrm{G}$ reported in the experiments is calculated from: 


$$
\begin{aligned}
& \text { R2 } \\
& \text { (r)2 rdr } \\
& G=\frac{R 1}{R 2} 2 r d r \\
& \text { Rl }
\end{aligned}
$$

A wide range of constant shear rate -1.4 to $138 \mathrm{~s}^{-1}(0.2$ to $20 \mathrm{rpm})$ and 0.7 to $40.7 \mathrm{~s}^{-1}(0.2$ to $12 \mathrm{rpm}$ ) for gap spacings of 1.5 and $3 \mathrm{~mm}$, respectively - in stable circular laminar flow conditions can be obtained with our device ( $=2 *$ rpm/60). Indeed, a laminar flow is created in the gap provided that the Taylor number Ta is less than $41^{26}$ :

$$
T a=\frac{e^{3 / 2} R^{1 / 2}}{2}
$$

where $\rho$ and $\eta$ are the density and the dynamic viscosity of the suspension $\left(\eta=1.08 \times 10^{-6} \mathrm{~m}^{2} . \mathrm{s}^{-}\right.$ ${ }^{1}$ ), e the gap spacing between cylinders, and $\mathrm{R}_{1}$ the radius of inner cyclinder. Maximum Taylor numbers of about 31 and 41 were attained in our experiments for gap spacings of 1.5 and $3 \mathrm{~mm}$, respectively.

At the beginning of each aggregation sequence, a shear gradient equal to $350 \mathrm{~s}^{-1}$ (i.e. the maximum shear rate possible with our set-up) was applied to the suspension for 3 min to break up the flocs already formed. A given angular velocity was then set and image acquisition was immediately started. Preliminary experiments revealed no dependence on the order of applied shear rates on floc formation, which is expected for reversible aggregation. However, close examination of the suspension revealed the presence of remaining aggregates after the breaking procedure. The mean diameter of those initial aggregates is plotted in figure 2 as a function of shear rate applied in the aggregation sequence following break-up. The average values of floc diameters are around 70 and $100 \mu \mathrm{m}$ for $2 \mu \mathrm{m}$ latex suspensions coagulated at $\phi=10^{-4}$ and $10^{-3}$, respectively, and they attain the $100-250 \mu \mathrm{m}$ and $175-300 \mu \mathrm{m}$ range for $0.2 \mu \mathrm{m}$ latex suspensions at particle volume fractions of $10^{-4}$ and $10^{-3}$, respectively. Obviously, a higher initial number concentration yields larger aggregates at the start of aggregation.

Therefore, our aggregating system may contain both elementary particles and flocs. The relative importance of shear aggregation to Brownian aggregation can be assessed by the Péclet number:

$$
P e=\frac{6 G d_{0}^{3}}{k T}
$$

where $d_{0}$ is the elementary particle radius, $k$ the Boltzmann constant, and $T$ the absolute temperature. The same expression is also frequently used for aggregates by substituting the 
aggregate radius of gyration for $\mathrm{d}_{0}{ }^{27}$. Péclet numbers above $4.410^{5}$ are thus obtained for flocs remaining after the breaking procedure. For latex particles with a $2 \mu \mathrm{m}$ diameter, Pe is in the 3.5-712 range. This suggests that, in comparison with shear-induced aggregation, the coagulation due to Brownian motion can be neglected for both initial flocs and $2 \mu \mathrm{m}$ latex particles. For $0.2 \mu \mathrm{m}$ particles, a Pe smaller than 1 is obtained at all shear rates which implies that the collisions due to Brownian motion become predominant in this aggregating system if those elementary particles are present. However, as our model is intended to describe the steady-state of a fully aggregated system, shear-induced aggregation is obviously the main mechanism at this stage.

Measurements of aggregate size and structure. Both cylinders of Couette reactor are made of transparent Altuglass ${ }^{\circledR}$. The outer cylinder is enclosed in a thermostated Altuglass ${ }^{\circledR}$ square box filled with water in order (i) to eliminate part of the dioptre effect and (ii) to control the temperature of the aggregating suspension (fig. 1c). All experiments were carried out at $30^{\circ} \mathrm{C}$. A Nd/YAG Q-switched laser was used to generate a pulsed laser sheet (about $1 \mathrm{~mm}$ in thickness) in the meridional plane of the gap, i.e. the stagnation plane (fig. 1d). The pulsed laser sheet was synchronised with a CCD camera (Kodak Megaplus ES1.0) to obtain a stroboscopic effect. The image capture frequency was fixed at $1 \mathrm{~Hz}$ for the first 100 seconds of shear aggregation (Dantec PIV2000 processor), and was then equal to $0.1 \mathrm{~Hz}$ until the end of the experiment. Images (1016x1008 pixels) were saved in a Tiff format. The resolution of the image is $140 \mu \mathrm{m}^{2} /$ pixel. The field of view was $12 \times 12 \mathrm{~mm}$. This corresponds to a $14^{\circ}$ arc that determines an inhomogeneous luminosity and a slight distortion effect for aggregates located on the image edges. As a result, both right and left image edges were clipped (about 100 pixels on each side) during image analysis. With this set-up, the aggregates out of focus are either not imaged or excluded during thresholding because of a higher gray level.

Images were first processed using Matlab 7.0 software. The raw gray-scale images were thresholded and converted to binary images (fig. 3a-b). Aggregates in contact with any image edge were erased. The aggregate characteristics (area A, maximum length $\mathrm{L}_{\max }$, aspect ratio, tilt angle with respect to the flow direction...) were then assessed with ImageJ software. The aspect ratio is defined from the ratio of major axis to minor axis of the ellipse that circumvents the aggregate, whereas the tilt angle corresponds to the angle between the primary axis of the best-fitting ellipse to the flow direction (x-axis of images) (fig. 3e). A circle equivalent diameter $\mathrm{D}$ was calculated from the projected area $\mathrm{A}$ of each floc: 
$D=2 \sqrt{A /}$. The size distribution was then obtained by counting the number of aggregates in logarithmically spaced size bins such that $d_{i+1}=2^{1 / 3} d_{i}$, which yields equally spaced size intervals on a $\mathrm{x}$-axis $\log$ scale (fig. $3 \mathrm{c}$ ). The mean aggregate size was calculated from $D(4,3)={ }_{i=1}^{i=k} n_{i} D_{i}^{4} /{ }_{i=1}^{i=k} n_{i} D_{i}^{3}$ with $n_{i}$ the number of flocs measured in range $\mathrm{i}, \mathrm{D}_{\mathrm{i}}$ the middle of class range, and $\mathrm{k}$ the total number of bins. The use of $\mathrm{D}(4,3)$ decreases the statistical weight of small aggregates which are not well resolved with our set-up.

The structure of aggregates was quantified from $A \sim L_{\max }{ }^{F_{2}}$ where $A$ is the projected area, $L_{\max }$ the maximum length, and $F_{2}$ the fractal dimension of the two-dimensional projection of aggregates. $F_{2}$ is equal to the $3 \mathrm{D}$ fractal dimension ${ }^{28,69} \mathrm{~F}$ provided that $\mathrm{F} \leq 2$. As illustrated in fig. 3f, the finite range of characteristic lengths imposed by our experimental set-up - image resolution of $40 \mu \mathrm{m}$ and gap spacing of 1500-3000 $\mu \mathrm{m}$ - limits the applicability of the power-law relationship to less than two decades in length, which is barely enough to define a true fractal dimension. Therefore, we will refer to the slope of the $\operatorname{Ln}(\mathrm{A})$ $\mathrm{Ln}\left(\mathrm{L}_{\max }\right)$ plot as the scaling exponent SE rather than the fractal dimension, hereafter.

\section{RESULTS AND DISCUSSION}

Time evolution of mean aggregate size. Typical evolutions of $\mathrm{D}(4,3)$ with time are shown in figure 4 for various shear rates, latex particle sizes of $0.2 \mu \mathrm{m}$ and $2 \mu \mathrm{m}$, and solid volume fractions of $10^{-3}$ and $10^{-4}$. In the literature, aggregation results are often reported using dimensionless parameters, i.e. a relative floc size versus a normalized number of collisions ${ }^{12,24,29}$. Such a representation was not adopted in fig. 4 as our primary objective was to determine a steady-state mean aggregate diameter. For suspensions coagulated from $2 \mu \mathrm{m}$ elementary particles at $\phi=10^{-3}$ (fig. 4a), the temporal evolution of average floc size exhibits a characteristic S-shape with an initial exponential growth phase which levels off to a steadystate average diameter ${ }^{12,24}$. This plateau size is markedly reduced and more rapidly attained with an increase in shear rate, as classically observed in shear-driven aggregation experiments $^{12}$. On the other hand, at a particle volume fraction of $10^{-4}$, a slight continuous increase in mean floc size is obvious especially at low shear gradients (fig. 4b). A longer acquisition time would have been required to reach a steady-state aggregation, though in that case, partial creaming of aggregates became noticeable.

The aggregation of $0.2 \mu \mathrm{m}$ latex particles leads to a slightly different pattern: the steady-state size (or close to) is larger and reached much faster for a given shear rate (fig. $4 \mathrm{c}$ - 
d). This behavior can be related to the higher initial number concentration of elementary particles in $0.2 \mu \mathrm{m}$ latex suspensions, i.e. $2.210^{17} / \mathrm{m}^{3}$ against $2.210^{14} / \mathrm{m}^{3}$ in the original $2 \mu \mathrm{m}$ latex suspensions for a particle volume concentration of $10^{-3}$. Indeed, the aggregation kinetics is, as a first approximation, second-order in destabilized particle/cluster concentration ${ }^{24,29}$. At very low shear gradients $\left(\mathrm{G}=3.7 \mathrm{~s}^{-1}\right.$ in fig. $\left.4 \mathrm{c}\right)$, it should be noted that the steady-state diameter of aggregates built up from $0.2 \mu \mathrm{m}$ particles at $\phi=10^{-3}$ is of the order of magnitude of the inner gap spacing of the Couette reactor. In that particular case, the examination of floc images revealed that most aggregates are oriented perpendicular to the flow direction (see fig. $8 \mathrm{~g}$ ), which may then identify a different aggregation regime.

Floc orientation in the shear flow. The preferential orientation of flocs mentioned above, is better evidenced from the distribution of tilt angles of aggregates to the flow direction. As illustrated in figure $5 \mathrm{c}$, the tilt angle shows a peak around $+80-85^{\circ}$ at $\mathrm{G}=3.7 \mathrm{~s}^{-1}$, whereas it decreases to about $\pm 10-15^{\circ}$, i.e. the flocs are aligned with the streamlines, at $G=88.6 \mathrm{~s}^{-1}$. In contrast, the aggregates formed from $2 \mu \mathrm{m}$ elementary particles yield a distribution of orientations centered around the horizontal for all shear gradients investigated (fig. 5a-b). The same trend, i.e. flocs generally aligned with the flow direction, was also found at lower particle volume fraction $\left(10^{-4}\right.$ and $\left.510^{-5}\right)$ for both 0.2 and $2 \mu \mathrm{m}$ coagulated suspensions (fig. 5d). Actually, aggregates mainly aligned perpendicular to the flow direction were only observed for $0.2 \mu \mathrm{m}$ elementary particles at a particle volume fraction of $10^{-3}$.

The distribution of tilt angles represents to some extent the time-averaged non-uniform rotary motion of aggregates in the shear flow. In our case, the time spent by the aggregates perpendicular to the shear direction is then much shorter than that aligned with the streamlines under most conditions. A common assumption in the literature is to consider flocs as solid ellipsoids ${ }^{6,9}$. Indeed, Veerapaneni and Wiesner showed that the flow passing through a porous aggregate drastically decreases with both floc size and fractal dimension ${ }^{30}$. Our flocs generally appear as oblate spheroids except for $0.2 \mu \mathrm{m}$ latex suspensions at $\phi=10^{-3}$ and $\mathrm{G}$ below $11.1 \mathrm{~s}^{-1}$ in which cases the aggregation regime leads to prolate spheroids. The rotation of a solid ellipsoid in a simple shear flow is strongly dependent on its initial position. However, a time-averaged period of rotation can be defined such that ${ }^{31}$ :

$$
\bar{T}=\frac{2}{G}\left(r_{e}+r_{e}^{1}\right) \text { with } r_{e}=\frac{2 a_{1}}{a_{2}+a_{3}}
$$

where $a_{1}, a_{2}$, and $a_{3}$, represent the equatorial radii and the polar radius of the ellipsoid. Therefore, the nearly identical distributions of floc orientations (fig. 5a) suggest that (i) the 
period of rotation of our aggregates is indeed inversely proportional to the shear rate, (ii) the flocs of coagulated latex particles behave similarly as solid ellipsoids in the shear flow. The average aspect ratio of latex aggregates - major to minor axis length ratio for the best-fit ellipse - was found to range from 1.4 to 1.8 (Tables in fig. 5), the upper value being observed for the higher shear rates. Assuming that our aggregates can be assimilated to spheroids, it can be calculated that $\bar{T} G$ varies between 13.3 and 14.8 , which is consistent with $\bar{T} G$ values close to 14 inferred from Blaser data ${ }^{6}$.

Aggregate size distributions. During floc growth, the number of aggregates contained in the captured images is too small to obtain a well-defined size distribution. Nevertheless, an average floc size $\mathrm{D}(4,3)$ can be calculated for each frame, and the size data replotted as a function of the reduced coordinate $\mathrm{D} / \mathrm{D}(4,3)$, where $\mathrm{D}$ is the circle equivalent diameter determined from the floc projected area. As illustrated in figure $6 \mathrm{a}-\mathrm{c}$ for $2 \mu \mathrm{m}$ latex suspensions coagulated under various shear rates at $\phi=10^{-3}$, the normalized aggregate size distributions collapse onto a time independent monomodal master curve. Such behavior, known as dynamic scaling ${ }^{32}$, is consistent with previous experimental, computer, and theoretical descriptions of aggregation processes involving both coagulation and break-up ${ }^{33-36}$. Similar self-preserving distributions can also be evidenced during the initial stages of aggregation for flocs of 0.2 and $2 \mu \mathrm{m}$ latex particles at $\phi=10^{-4}$ (fig. 6d-f). As mentioned above, suspensions of $0.2 \mu \mathrm{m}$ particles coagulated at a volume fraction of $10^{-3}$ reach the steady-state too rapidly to enable the observation of self-similar distributions. The scaling distributions exhibit a characteristic tail toward the small floc sizes that becomes less extended upon lowering either the initial particle volume fraction or the shear gradient. The latter effect, rather unintuitive at first since an increase in shear rate is expected to generate a higher number of floc fragments and hence to yield a broader distribution, becomes trivial if the size range of aggregates that makes up the distribution starts at the primary particle unit. Narrower distributions with an increase in shear rate were also reported by Kikuchi et al. ${ }^{37}$ for latex particles coagulated between two parallel plates.

At the steady-state, in order to provide sufficient statistics, all floc images recorded under given conditions were used to derive the corresponding aggregate size distribution. It was first verified that a steady-state floc size distribution was indeed achieved by averaging floc data at different time intervals, although, as pointed out by Topelkin et al. ${ }^{38}$, such operation does not ensure that the coagulated suspension has attained a rigorous stationary 
state. The distributions thus obtained were nearly undistinguishable in the particle size range investigated for suspensions coagulated at a particle volume fraction of $10^{-3}$. On the other hand, at $\phi=10^{-4}$, as can be expected from the time evolutions of mean floc size shown in fig. 4, a slight - although minor - shift was detected between the time-averaged distributions especially at small shear gradients (see the supporting information material). Still, the main features of those time-averaged floc size distributions were not found to vary significantly and they were then assumed to be representative of steady-state.

Figure 7 shows typical time-averaged floc size distributions at - or close to - the steady-state as a function of shear rate for aggregates of $0.2 \mu \mathrm{m}$ and $2 \mu \mathrm{m}$ and particle volume fractions of $10^{-4}$ and $10^{-3}$. The shape of most distributions is reminiscent of that observed during floc growth, i.e. monomodal with a more or less pronounced skewness toward the smaller aggregate sizes. At $\phi=10^{-4}$ and high shear levels, the tail of the size distribution is truncated because the minimum particle size detection threshold is reached. Interestingly, the distributions are broader at both low and high shear rates, and even bimodal at very low shear gradients. Floc images taken at the steady-state under those conditions (fig. 8a-d-g-j) clearly corroborate the presence of such bimodal patterns. At intermediate shear gradients, truly monodisperse aggregates are obtained, that then rearrange as a function of time to yield an approximate 2-dimension body-centered cubic organization (fig. 8h-k). In the framework of a dynamic aggregation process, such organization is reminiscent of a Turing pattern. However, as the flocs of coagulated latex particles behave in the shear flow similarly as solid ellipsoids, the observed arrangement may also simply originate from the relative influence of wake regions behind the aggregates.

Such results are in contrast with most previous studies on this topic. Indeed, the experimental aggregate size distributions reported in the literature are generally monomodal for a large range of model, natural, and engineered systems ${ }^{35,38-40,}$ even though destabilized suspensions of casein micelles ${ }^{42}$, bloc copolymer colloids ${ }^{43}$, and aggregated polystyrene sulfate latex ${ }^{44}$ have been shown to yield multimodal patterns under certain conditions. As aggregation is more conveniently monitored by using a moment derived from the floc size distribution rather than the distribution itself, e.g. a mean aggregate size determined from the light scattered by the destabilized suspension, the presence of multimodal aggregates may have been overlooked in some cases. Theoretical and experimental investigations of steadystate floc size distributions obtained when simultaneous coagulation and breakup occur, have also revealed that those distributions should be self-preserving with respect to shear rate ${ }^{34-}$ 36,45 . In our case, the observation of both monomodal and bimodal stable distributions at 
various shear rates precludes such scaling. Nevertheless, although unusual, the occurrence of bimodal distributions at the steady-state remains within the framework of reversible aggregation. Analytical evidence of such behavior has been provided by Vigil and Ziff ${ }^{33}$ for particular forms of coagulation and fragmentation kernels, and more recently, such stable bimodal patterns have been related with a predominant erosion mode ${ }^{36}$. The shift with shear rate of steady-state distributions from bimodal to narrow monomodal and then to broader monomodal, might then indicate a change in the nature of breakage mode according to the shear force exerted onto the floc.

Comparison of self-preserving distributions established during floc growth with the corresponding bimodal steady-state distributions (see fig. 6-7), reveals that the monomodal form evolves towards a bimodal pattern at the end of the growth period. Such observation is consistent with previous work by Soos et al. ${ }^{46}$ about the change in shape of floc size distributions during aggregation. It also fits the classical scheme of an aggregate size initially controlled by a dominant shear aggregation, and upon reaching the steady-state, by an enhanced floc breakup ${ }^{47}$. However, according to Brakalov ${ }^{25}$, the stable average floc size could also result from a decrease in collision efficiency. In other terms, the steady-state aggregates would then correspond to hydrodynamically isolated entities and not to flocs undergoing simultaneous aggregation and breakup. The formation at low shear rate of a stable bimodal floc size distribution following a monomodal growth regime appears as a definite experimental evidence of such dynamic equilibrium.

Floc structure. Figure 9 displays the temporal evolution of scaling exponent characterizing the floc structure under experimental conditions identical to those described in figure 4 . For all coagulated suspensions, SE closely follows the variation of mean aggregate size with time. The initial value of scaling exponent is around 1.6 and it remains close to that value at elevated shear rates for which no supplementary floc growth can be detected. At low and intermediate shear gradients, SE increases moderately to about $1.8 \pm 0.1$. Such range of values, consistent with a diffusion-limited cluster aggregation mechanism ${ }^{28}$, has been observed both experimentally and numerically for shear-induced aggregation in laminar flow at relatively low volume fractions ${ }^{48-51}$. The scaling exponent of 1.6 is measured for aggregates resulting from the breaking procedure taking place before the aggregation sequence: according to Le Berre et al. ${ }^{50}$, such lower value might be attributed to a predominant rotational effect during floc formation, although it might also simply be ascribed to the less accurate determination of SE from smaller projected flocs ${ }^{52}$. 
A rearrangement of aggregates to more compact fractal structures is frequently evidenced when flocs are exposed to shear ${ }^{12,24,29}$. Similarly to aggregate breakup, the amount of restructuring can be related to the interplay between the bonding forces that hold elementary particles within the floc and the hydrodynamic shear stresses applied onto the aggregate. Typically for electrolyte-induced aggregation, flocs built up from small particles are more easily restructured because of weaker van der Waals interactions between the destabilized particles, whereas aggregates composed of larger primary particles are held by stronger and hence more rigid bonds, which render them more prone to fragmentation under shear stresses ${ }^{29}$. In our case, the aggregates formed from either 0.2 or $2 \mu \mathrm{m}$ latex particles are characterized by the same scaling exponent close to 1.8 over a large range of shear rates (fig. 10). Taking into account previous literature ${ }^{48,49,53,54}$, this suggests at first sight that (i) both types of latex particles establish sticky bonds within the floc, (ii) aggregation should proceed on the basis of one or two contact points between colliding flocs, a fractal dimension of 1.8 corresponding to a 2.2 nearest neighbours per particle, and (iii) restructuring can be neglected for the two systems at the aggregate size scale.

Data fitting. The co-existence of both bimodal and monomodal distributions of varying broadness at the steady-state, prevents from using the mean aggregate size as a maximum size criterion. Instead, the mode $\mathrm{D}_{\mathrm{m}}$ of the largest floc population appears more relevant since, in the framework of investigating the cohesiveness of aggregates, the hydrodynamic forces that are applied onto the floc roughly increases with the square of the aggregate size. The dependence of $D_{m}$ versus shear rate is plotted on a log-log scale in figure 11 for the aggregates built up from 0.2 or $2 \mu \mathrm{m}$ latex sizes at all particle volume fractions investigated in this study. The solid lines on the graphs represent the least-square regression fits of the experimental data to equation (5), thus showing that the data are nicely described by our model. The influence of particle volume fraction on steady-state aggregate size is in general accordance with previous results of the literature ${ }^{13,24}$, i.e. (i) the stable floc diameter increases with $\phi$ and more significantly at low shear rates, (ii) the dependence on shear rate of the steady-state aggregate size strongly attenuates below a critical particle volume fraction (e.g. 5 $10^{-5}$ for $0.2 \mu \mathrm{m}$ primary particle size). It should also be noted that the change in width of floc size distributions with shear rate noted above, although not predicted by our model, seems quite in line with the decrease of aggregate size in two successive stages.

However, as illustrated in figure 12 , the evolution of mean aggregate diameter $\mathrm{D}(4,3)$ with shear rate is also adequately described by the classical power law relationship $D=\alpha G^{-\beta}$. 
Moreover, the average slopes of the $\log \mathrm{D}(4,3)-\log \mathrm{G}$ plots, -0.26 and -0.42 for 0.2 and $2 \mu \mathrm{m}$ coagulated latex, respectively, are in good agreement with some experimental data reported in the literature ${ }^{12,19,21,23}$. In addition, a theoretical basis for those particular values has been provided by Potanin $^{22}$ : thus in the framework of pure breakup events, an exponent equal to 0.42 identifies central interactions between neighboring particles within the floc, whereas the slope of -0.26 can be related to the contribution of a supplementary non-central component such as surface roughness in the two-particle interaction. Still, in most previous studies where a dynamic equilibrium between floc growth and floc breakup is suspected, a slight deviation of experimental results from the power law can generally be detected, the mean aggregate size being most often overpredicted at low shear rate and underestimated at high shear rate $^{12,19,21,55}$.

The sum of two exponential terms appears to reconcile the differing views about the main cause limiting the growth of aggregates under shear ${ }^{20,25}$ : indeed, the relative positions on the $x$-axis of critical shear gradients of floc formation $G_{f}$ and of aggregate dislocation $G_{d}$ (fig. 11 ), imply that the maximum aggregate size is principally controlled by breakup at $G \geq G_{d}$, and that it is also governed by a decrease in capture efficiency at higher shear $\left(G \geq G_{f}\right)$. The narrowest size distributions are observed in the dip of the curve fit $\left(G_{d} \leq G \leq G_{f}\right)$, where both floc growth and breakup should concur in equivalent manner for determining the limiting floc size. It is also worth noting that the decrease in SE observed at elevated shear rates occurs precisely beyond $G_{f}$ (see fig. 10), thus revealing the dominant influence of the original fractal organization on the final floc structure.

Significance of model parameters. The values of $G_{f}, G_{d}, D\left(G_{f}\right), D\left(G_{d}\right)$, and $D_{\max }$, derived from the regression curves displayed in fig. 11 are given in table 1 . The floc cohesive force $F_{C}$ is generally evaluated from the maximum hydrodynamic force $\mathrm{F}_{\mathrm{H}}$ acting on the aggregate upon breakage $e^{6,22,46}$. Adopting a common assumption in the literature - although conceptually arguable - namely that a floc surface on which $\mathrm{F}_{\mathrm{H}}$ is applied can be defined for a mass fractal aggregate, $\mathrm{F}_{\mathrm{H}}$ can be expressed by: ${ }^{6,21}$

$$
\mathrm{F}_{\mathrm{H}} \sim \mu \mathrm{G}_{\mathrm{d}} \mathrm{D}\left(\mathrm{G}_{\mathrm{d}}\right)^{2}
$$

Even under pure shear flow, the calculation of hydrodynamic load exerted on a triaxial ellipsoid is far from trivial as it changes with time during the particle rotation ${ }^{56}$. To simplify, we assimilate the aggregate to a sphere, and the maximum drag on each hemisphere is then equal to: ${ }^{57}$ 


$$
F_{C}=\frac{5}{8} D\left(G_{d}\right)^{2} \quad G_{d}
$$

Figure 13 shows the estimated values of $F_{C}$ as a function of $D\left(G_{d}\right)$. The set of rupture forces determined by Blaser ${ }^{6}$ for silica/ferric hydroxide aggregates subjected to a plane Couette flow, is also displayed on the same graph. Interestingly, even though the destabilization mechanisms underlying the formation of the two types of aggregates considered in fig. 13 are certainly different, $\mathrm{F}_{\mathrm{c}}$ scales as $\mathrm{D}^{3 / 2}$ in both cases. Such a result, although disagreeing with the broadly accepted view that larger flocs should be more fragile than smaller aggregates ${ }^{23}$, is consistent with several other experimental reports indicating an increase of the aggregate cohesive force with floc size $e^{6,10,11}$. Nevertheless, in the case of aggregates formed from soil particles coagulated with $\mathrm{NaCl}, \mathrm{CaCl}_{2}$, or $\mathrm{HCl}$, Kobayashi ${ }^{67}$ found that the cohesive force remained constant in the $10-50 \mu \mathrm{m}$ size range. It is then possible that, below a characteristic aggregate size, the floc cohesive force becomes constant and roughly equal to the bonding force between two primary particles.

The $3 / 2$ exponent can be derived from a simple description of the floc deformation in the flow field before rupturing. Let $\mathrm{E}$ be the elastic modulus of the aggregate, $\mathrm{D}$ an initial characteristic length, and $\Delta$ an infinitesimal displacement of the floc (see inset in fig. 13). $\Delta$ is related to D through:

$$
=D(1 \quad \cos \quad) \approx D \frac{2}{2} \text { and hence: } \quad \approx\left(\frac{2}{D}\right)^{\frac{1}{2}}
$$

The shear stress is expressed as: $x y=E \quad \frac{F_{C}}{D^{2}}$

Then, from the two previous equations: $F_{C}=\int_{x y} d S \approx E\left(\frac{2}{D}\right)^{\frac{1}{2}} D^{2}$ which leads to $F_{C} \quad D^{1.5}$. It is however questionable whether the elastic modulus of the aggregate is independent of floc size $\mathrm{e}^{58}$.

The values of aggregate cohesive forces determined in this study range from $1.64 \mathrm{nN}$ to about $38 \mathrm{nN}$, which is comparable with direct and indirect measurements of aggregate rupture forces or adhesion forces previously reported ${ }^{2,6,9,46,59,60}$. In the literature, the floc cohesive force is often identified to $\mathrm{F}_{\mathrm{C}}=f \mathrm{~N}_{\mathrm{C}}$, where $f$ represents the bonding force between two primary particles and $\mathrm{N}_{\mathrm{C}}$ the number of contacts in the original aggregate between the daughter flocs generated by a breakup event ${ }^{61}$. In addition, for suspensions coagulated with a high electrolyte concentration, the double-layer repulsion is expected to be completely 
suppressed, and it is then assumed that van der Waals forces represent the main attractive contribution between primary particles. Therefore, for two identical spheres of radius $r$ separated by a minimum distance $\delta$ with $\delta<r$ r:

$$
f=\frac{A_{H} r}{12^{2}}
$$

where $A_{H}$ is the Hamaker constant. A typical value for $\delta$ is three water layers, i.e. $\delta=0.75$ nm. Using Lifshitz theory, Prieve and Russel ${ }^{62}$ calculated a Hamaker constant equal to 1.37 $10^{-20} \mathrm{~J}$ for latex particles in aqueous systems. However, it has been argued that in the presence of a high salt concentration, $A_{H}$ should be reduced to about $110^{-21} \mathrm{~J}$ to adequately describe the aggregation of latex particles ${ }^{51}$.

In the case of $2 \mu \mathrm{m}$ primary particles, these two values of Hamaker constant yield $f=$ $0.14 \mathrm{nN}$ and $f=2 \mathrm{nN}$, respectively. Obviously, the $2 \mathrm{nN}$ value, i.e. $\mathrm{A}_{\mathrm{H}}$ equal to $1.3710^{-20} \mathrm{~J}$, is directly comparable with the magnitude found for the cohesive force of aggregates built-up from $2 \mu \mathrm{m}$ latex particles. In other words, the number of connecting bonds $\mathrm{N}_{\mathrm{C}}$ that should be broken to release two daughter flocs, should be comprised between 1 to 3, which is consistent with previous experimental results and aggregate breakup models reported in the literature ${ }^{22,48,61}$. A $N_{C}$ value greater than 1 implies that some structural readjustments have occurred upon aggregate-aggregate association such as rotation about the contacting particle until a second or even a third connecting bond is established between the colliding flocs ${ }^{63}$.

The much higher $\mathrm{N}_{\mathrm{C}}$ values - 17 to 185 - found for the smaller latex size might be attributed to an increased compactness of aggregates that would occur at length scales smaller than those investigated with our set up (image resolution of $40 \mu \mathrm{m}$ ). Indeed, for a given shear environment, a higher fractal dimension is usually determined for flocs formed from smaller elementary particles ${ }^{29}$. In agitated suspensions, it is also common to observe an increasing fractal dimension during aggregation $1^{12,24,29,64,65}$. When the floc structure is characterized by light scattering, the presence at the early growth stages of remaining primary particles and of small clusters certainly affects the determination of fractal exponent ${ }^{24}$. Still, that larger flocs are more compact than smaller aggregates is quite in line with the increase in cohesive force with floc size observed above ${ }^{12,64}$. The length scale of increased floc compactness should then vary with the initial number of primary particles.

Instead of reaching an infinite size, the aggregate size levels off to a maximum diameter when the shear rate tends towards zero (fig. 12). As illustrated in figure 14a, the dimensionless maximum aggregate diameter $D_{\max } / \mathrm{D}_{\mathrm{o}}$ is proportional to the initial number of primary particles on a log-log scale, which suggests that the total number of aggregates 
formed in the Couette reactor is a constant. Perhaps less surprising but not predicted by our model, figure $14 \mathrm{~b}$ indicates that the critical shear gradients for floc breakup and for floc formation are linearly correlated for a given primary particle size. In other terms, the manner by which the floc is built-up from small clusters and primary latex particles, is close to the manner it can be disassembled. These two aspects remain to be investigated in details.

Effect of Couette reactor geometry on aggregation results. Most investigations of particle aggregation using a Couette-flow system are performed with a rotating inner cylinder and a stationary outer cylinder ${ }^{13,29,66}$. In that case, the aggregates are dragged with the flow shear which ensures a continuous renewal of the coagulated suspension in the field of view. In contrast, by rotating the cylinders in opposite directions at equal angular velocity, a stagnation plane is created in the middle of the annular gap. However, the aggregates formed do not remain at the same location within that plane: because of the shear profile and of their irregular shape, they are observed to rotate and, from time to time, to tumble outside the field of view upon collision with moving flocs. The renewal of aggregates within the field of view is nevertheless much slower than in the classical Couette reactor at a given average shear rate. Actually, the evolution of average floc diameter with time showed a similar scatter of data points for both configurations of Couette reactor (see the supporting information material). Interestingly, when both cylinders rotate in opposite directions at low shear rate $\left(\mathrm{G} \leq 10.3 \mathrm{~s}^{-1}\right)$, the steady-state mean aggregate size is slightly shifted to smaller sizes even though the scaling exponents characterizing the structure of aggregates are equivalent for both flow systems (SE 1.9), whereas, at higher shear rate, the opposite pattern is observed (fig. 15a). Therefore, the hydrodynamic environment experienced by the aggregates is slightly different according to the configuration selected for the Couette reactor. That the same average shear gradient may yield a different steady-state floc size according to the hydrodynamic flow field developed in a reactor, is a well-established result in chemical engineering for turbulent agitation $^{35}$.

A change in the inner gap spacing of Couette reactor may also affect the aggregation process $^{37}$. Indeed, figure $15 \mathrm{~b}$ reveals that a decrease in the degree of geometrical confinement, i.e. a larger gap spacing, significantly modifies the $G_{f}$ and $G_{d}$ values but not the limiting aggregation regime characterized by $\mathrm{D}_{\max }$. In other words, the formation of aggregates is promoted at low shear rates with a larger gap spacing, whereas it becomes clearly hindered at higher shear gradients in comparison with the confined configuration. The presence of walls should influence many aspects of the aggregation process, such as the trajectories of the 
colliding flocs, the rotational speed of individual aggregates, and the drainage rate of fluid between aggregating units. Furthermore, as indicated in the inset fig. 15b, although the shear rate experienced by the aggregates is equivalent in both situations, the absolute fluid velocity is slightly lower in the case of an increased degree of confinement. A thorough analysis of all these effects upon floc growth and floc breakup is nevertheless beyond the scope of this paper.

\section{Concluding remarks:}

According to our results, the power law relationship classically used in the literature to describe the maximum stable floc size as a function of shear rate, is not valid when a large range of shear conditions is investigated. Instead, a sum of two exponential laws is necessary to adjust the experimental data. Such evolution can be modeled by taking into acount both floc formation and floc breakup in the definition of the steady-state aggregate size. The model introduces a critical shear gradient of aggregate dislocation that is used to calculate the floc cohesive force. In contrast to the accepted view of larger flocs more fragile than smaller ones, but in accordance with previous measurements of floc binding strength reported in the literature, we find that the floc strength increases with aggregate size beyond a given floc size. The origin of such increase should be investigated, to the least by providing a better description of aggregate structure at various length scales.

Two other experimental systems - latex particles coated with a polymer of tunable hydrophobicity and natural humic substances coagulated either with ferric chloride or $\mathrm{Al}_{13}$ polycations - have been investigated. All the results obtained corroborate those reported in this publication and are the subject of two forthcoming papers. Data sets from the literature acquired under conditions of turbulent aggregation, were also successfully adjusted by a sum of two exponential laws. An improved aggregation model is nevertheless necessary to explain (i) the formation of multimodal floc populations, (ii) the effect of particle volume fraction on floc size, (iii) the influence of geometrical confinement on the equilibrium aggregate size. At steady-state, it would also be interesting to precise the size distribution of aggregated units exchanged between flocs and the frequency of such exchanges.

Acknowledgements : Financial support provided by CNRS-GDR PROSURF is gratefully acknowledged by the authors

Supporting Information Material: Figures S1 and S2 show the floc size distributions averaged at different time intervals at steady-state for aggregates formed with latex particle 
size of 0.2 and $2 \mu \mathrm{m}$ and at particle volume fractions of $10^{-4}$ and $10^{-3}$. Figure S3 presents various temporal evolutions of $\mathrm{D}(4,3)$ for the two configurations of Couette reactor investigated in this study. Figure S4 shows the aggregation results obtained with a gap spacing of $3 \mathrm{~mm}$ in the case of aggregates formed with $0.2 \mu \mathrm{m}$ primary particles at a $10^{-4}$ volume fraction. This information is available free of charge via the Internet at http://pubs.acs.org/.

\section{References}

(1) Bache, D.H., Gregory, R. Flocs in water treatment. IWA publishing, 2007.

(2) Yeung, A.K.C., Pelton, R. J. Colloid and Interface Sci. 1996, 184, 579-585.

(3) Yeung, A.K.C., Gibbs, A., Pelton, R. J. Colloid Interface Sci. 1997, 196, 113-115.

(4) Kao, S.V., Mason, S.G. Nature 1975, 253, 619-621.

(5) Pandya, J.D., Spielman, L.A. J. Colloid Interface Sci. 1982, 90(2), 517-531.

(6) Blaser, S. J. Colloid Interface Sci. 2000, 225, 273-284.

(7) Sonntag, R.C., Russel, W.B. J. Colloid Interface Sci. 1987, 115(2), 390-395.

(8) Higashitani, K., Inada, N., Ochi, T. Colloids and Surf. 1991, 56, 13-23.

(9) Kobayashi M. Colloids and Surfaces A: Physicochemical and Engineering Aspects 2004, 235, 73-78

(10) Glascow, L.A., Ping Hsu J.Y.H. AIChE J. 1982, 28(5), 779-785.

(11) Bache, D.H., Al-Ani, S.H. Wat. Sci. Tech. 1989, 21, 529-537.

(12) Oles, V. J. Colloid Interface Sci. 1992, 154, 351-358.

(13) Serra, T., Colomer, J., Casamitjana, X. J. Colloid Interface Sci. 1997, 187, 466-473.

(14) Tomi, D.T., Bagster, D.F. Trans. IchemE 1978, 56, 9-18.

(15) Gregory, J. Colloids Surf. 1988, 31, 231-253.

(16) François, R.J., Wat. Res. 1987, 21, 1023-1027.

(17) Jarvis P. , Jefferson B., Gregory J., Parsons S.A. Wat. Res, 2005, 39, 3121-3137

(18) Parker, D.S., Kaufman, W.J., Jenkins, J. Pro ASCE. Journal of the Sanitary Engineering Division 98(SA1), 1972, 79-99.

(19) Hunter, R.J., Frayne, J. J. Colloid Interface Sci. 1980, 76(1), 107-115.

(20) Tambo, N., Hozumi, H. Wat. Res. 1979, 13, 421-427. 
(21) Sonntag, R.C., Russel, W.B. J. Colloid Interface Sci. 1986, 113, 399-413.

(22) Potanin, A.A. J. Colloid Interface Sci. 1993, 157, 399-410.

(23) Bache, D.H. Chem. Engng. Sci. 2004, 59, 2521-2534.

(24) Moussa, A.S., Soos, M., Sefcik, J., Morbidelli, M. Langmuir 2007, 23, 1664-1673.

(25) Brakalov, L.B. Chem. Engng. Sci. 1987, 42(10) 2373-2383.

(26) Guyon, E., Hulin, J.P., Petit, L. Hydrodynamique physique. EDP Sciences, CNRS Editions. 2001

(27) Mokhtari, T., Chakrabarti, A., Sorensen, C.M., Cheng, C.Y., Vigil, D. J. Colloid Interface Sci. 2008, 327, 216-223.

(28) Meakin, P. Adv. Colloid Interface Sci. 1998, 28, 249-331.

(29) Selomulya C., Bushell G., Amal R., and Waite T. D. Langmuir, 2002, 18, 1974-1984

(30) Veerapaneni, S., Wiesner, M.R., J. Colloid Interface Sci. 1996, 177, 45-57.

(31) Van de Ven, T.G.M. Colloidal Hydrodynamics. Academic Press, London. 1989

(32) Friedlander S.K. Smoke, Dust and Haze, Wiley, 1977

(33) Vigil, R.D., and Ziff, R.M. J. Colloid Interface Sci. 1989, 133(1), 257-264.

(34) Pefferkorn, E., Stoll S., J. Colloid Interface Sci. 1990, 138(1), 261-272.

(35) Spicer, P.T. and Pratsinis, S.E. AIChE Journal 1996, 42(6) 1612-1620.

(36) Babler, M.U. and Morbidelli M. J. Colloid Interface Sci. 2007, 316, 428-441.

(37) Kikuchi, Y., Yamada, H., Kunimori, H., Tsukuda, T., Hozawa, M., Yokoyama, C. Kubo, M. Langmuir 2005, 21, 3273-3278.

(38) Topelkin, V.A., Duits, M.H.G., van den Ende, D., Mellema, J. Langmuir 2004, 20, 26142627.

(39) Hunt, J.R. J. Fluid. Mech. 1982, 122, 169-185.

(40) Lartiges, B.S., Deneux-Mustin, S., Villemin, G., Mustin, C., Barres, O., Chamerois, M., Gerard, B., Babut, M. Wat. Res. 2001, 35(3), 808-816.

(41) Chaignon, V., Lartiges, B.S., El Samrani, A., Mustin, C. Wat. Res. 2002, 36(3), 676-684.

(42) Chu B., Zhou Z., Wu G.,. Farrell Jr. H.M J. Colloid and Interface Sci, 1995, 170, 102112 
(43) Wu G., Chu B., Schneider D.K. J. Phys. Chem. 1995, 99, 5094-5101

(44) Widmaier, J., and Pefferkorn, E. J. Colloid and Interface Sci. 1998, 203, 402-418.

(45) Family, F., Meakin, P., Deutch, J.M. Phys. Rev. Lett. 1986, 57, 727-730.

(46) Soos, M., Moussa, A.S., Ehrl, L., Sefcik, J., Wu, H., Morbidelli, M. J. Colloid Interface Sci. 2008, 319, 577-589.

(47) Soos, M., Sefcik, J. Morbidelli, M Ind. Eng. Chem. Res. 2007, 46, 1709-1720.

(48) Torres, F.E., Russel, W.B., Schowalter, W.R. J. Colloid Interface Sci. 1991a, 142(2), 554-574.

(49) Torres, F.E., Russel, W.B., Schowalter, W.R. J. Colloid Interface Sci. 1991b, 145(1), 5173.

(50) Le Berre, F., Chauveteau, G., Pefferkorn, E. J. Colloid Interface Sci. 1998, 199, 13-21.

(51) Sato, D., Kobayashi, M., Adachi, Y. J. Colloid Interface Sci. 2004, 272, 345-351.

(52) Adachi, Y., Kobayashi, M. Ooi, S. J. Colloid Interface Sci. 1998, 208, 353-355.

(53) Adachi, Y. Ooi, S. J. Colloid Interface Sci. 1990, 135(2), 374-384.

(54) Potanin, A.A. J. Colloid Interface Sci. 1991, 145, 140-157.

(55) Boller, M., Blaser, S. Wat. Sci. Tech. 1998, 37(10), 9-29.

(56) Blaser, S. Chem. Eng. Sci. 2002, 57, 515-526.

(57) Coufort, C., Liné, A. Chem. Eng. Res. and Design 2003, 81, 1206-1211.

(58) Buscall, R., McGowan, I.J., Mills, P.D.A., Stewart, R.F., Sutton, D., White, L.R., Yates, G.E. J. Non-Newtonian Fluid Mech. 1987, 24, 183-202.

(59) Hodges C.S., Looi L, Cleaver J.A.S., and Ghadiri M. Langmuir 2004, 20, 9571-9576

(60) Jaiswal, R.P., Kumar G., Kilroy, C.M. Beaudoin, S.P. Langmuir 2009, 25(18), 1061210623.

(61) Kobayashi, M., Adachi, Y., Ooi, S. Langmuir 1999, 15, 4351-4356.

(62) Prieve D.C. , Russel W.B J. Colloid Interface Sci. 1988, 125, 1-13

(63) Meakin, P., Jullien R. J. Phys. 1985, 46(9) 1543-1552.

(64) Lin, M.Y., Klein, R., Lindsay, H.M., Weitz, D.A., Ball, R.C., Meakin, P. J. Colloid Interface Sci. 1990, 137(1), 263-280. 
(65) Serra, T., Casamitjana, X. J. Colloid Interface Sci. 1998, 206, 505-511.

(66) Wang, L., Marchisio, D.L., Vigil, R.D., Fox, R.O. J. Colloid Interface Sci. 2005, 282, 380-396.

(67) Kobayashi, M. Wat. Res. 2005, 39, 3273-3278.

(68) Soos, M., Ehrl, L., Bäbler, M.U., Morbidelli, M. Langmuir 2010, 26(1), 10-18.

(69) Jullien, R., and Botet, R., 1987, Aggregation and Fractal Aggregates, World Scientific Ed., Singapore. 


\begin{tabular}{|c|c|c|c|c|c|}
\hline $\mathbf{d}_{\mathbf{0}}(\boldsymbol{\mu m})$ & 2.0 & 2.0 & 0.2 & 0.2 & 0.2 \\
\hline $\boldsymbol{\Phi}$ & $10^{-3}$ & $10^{-4}$ & $10^{-3}$ & $10^{-4}$ & $5.10^{-5}$ \\
\hline $\mathbf{G}_{\mathbf{d}}\left(\mathbf{s}^{-\mathbf{1}}\right)$ & 3.6 & 7.4 & 2.4 & 3.4 & 5.5 \\
\hline $\mathbf{G}_{\mathbf{f}}\left(\mathbf{s}^{-\mathbf{1}}\right)$ & 20.7 & 43.3 & 17.4 & 36.2 & 47.7 \\
\hline $\mathbf{D}\left(\mathbf{G}_{\mathbf{d}}\right)(\boldsymbol{\mu m})$ & 883.7 & 410.1 & 2642.9 & 1391.8 & 528.7 \\
\hline $\mathbf{D}\left(\mathbf{G}_{\mathbf{f}}\right)(\boldsymbol{\mu m})$ & 424.4 & 222.2 & 712.8 & 441.9 & 359.3 \\
\hline $\mathbf{D}_{\mathbf{m a x}}(\boldsymbol{\mu m})$ & 1546.3 & 671.0 & 5731.0 & 2939.3 & 753 \\
\hline
\end{tabular}

Table 1: Values of $G_{f}, G_{d}, D\left(G_{f}\right), D\left(G_{d}\right)$, and $D_{\max }$, derived from the regression curves displayed in fig. 11 (gap spacing=1.5 mm). 